\title{
Functional Outcomes of Uni-knee Arthroplasty for Medial Compartment Knee Arthropathy in Asian Patients
}

\author{
To Wong ${ }^{1}$, Ching-Jen Wang ${ }^{2}$, Jun-Wen Wang², Jih-Yang Ko
}

Background: Uni-knee arthroplasty (UKA) has shown better knee kinematics and motion that may better suit the activities of daily living in Eastern countries. The purpose of this study was to evaluate the functional outcomes of UKA for medial compartment knee arthropathy in Asian patients.

Methods: The study cohort consisted of 48 patients with 51 UK A knees. Only one type of prosthesis was used and all components were cemented. Postoperative management included ambulation with weight bearing, range of motion, and muscle strengthening exercises as tolerated until full recovery. The average follow-up was $52.0 \pm 24.0$ (range 12-92) months. The evaluation included functional assessment, the Knee Society knee and functional scores, the International Knee Document Committee (IKDC) subjective and objective scores, and radiographs of the knee.

Results: $\quad$ The overall clinical outcomes of the knee showed the functional outcome of the knee to be normal in $51 \%$, nearly normal in $37 \%$, abnormal in $8 \%$, and severely

\section{At a Glance Commentary}

Scientific background of the subject

Uni-knee arthroplasty (UKA) is one of the surgical option for medial compartment knee arthropathy include osteoarthritis or osteonecrosis. Functional outcomes of UKA had been discussed among the western population. Better knee kinematics and motion in UKA design that consider more appropriate for the activities of daily living in Eastern countries.

\section{What this study adds to the field}

This study showed that UKA provides excellent pain relief and restoration knee daily activities that perfectly fit the oriental lifestyle and high patient satisfaction in Asian patients at medium-term follow-up. Low complications rate and good survival rate were found. abnormal in $4 \%$. The functional activities included stair climbing in $96 \%$, squatting in $76 \%$, jogging in $71 \%$ and kneeling in $47 \%$. Three-quarters of the patients were able to kneel for daily activities. Approximately $98 \%$ of the patients were satisfied with the operation. The survivorship of the prosthesis was $98 \%$ with one revision pending. Radiographic evaluations revealed the components were centered in $82 \%$ and off-centered in $18 \%$. Osteoarthritis was $22 \%$ preoperative and $27 \%$ postoperative for the patellofemoral compartment, and $0 \%$ before and $4 \%$ after surgery for the lateral compartment. The functional outcomes showed no difference between patients with and without patellofemoral arthritis. The complications included one component malposition and one knee pain of undetermined origin.

Conclusions: UKA provides excellent pain relief and restoration of knee function including kneeling, squatting, and sit-to-stand activities that perfectly fit the oriental lifestyle and high patient satisfaction in Asian patients at medium-term follow-up. The complications were rare and the survival rate was $98 \%$ at medium-term follow-up.

(Biomed J 2014;37:406-410)

Key words: Asian, medial compartment knee arthropathy, uni-knee arthroplasty

From the ${ }^{1}$ Department of Orthopedic Surgery, Kaohsiung Municipal United Hospital, Kaohsiung, Taiwan; ${ }^{2}$ Department of Orthopedic Surgery, Kaohsiung Chang Gung Memorial Hospital and Chang Gung University College of Medicine, Kaohsiung, Taiwan Received: Jan. 25, 2013; Accepted: Jan. 15, 2014

Correspondence to: Dr. Ching-Jen Wang, Department of Orthopedic Surgery, Kaohsiung Chang Gung Memorial Hospital. 123, Tapei Rd., Niaosung, Kaohsiung 833, Taiwan (ROC). Tel: 886-7-7335279; Fax: 886-7-7335515; E-mail: w281211@adm.cgmh.org.tw

DOI: $10.4103 / 2319-4170.132877$ 
$\mathrm{T}$ The surgical management options for medial compartment knee arthropathy (osteoarthritis or osteonecrosis) include uni-knee arthroplasty (UKA), total knee arthroplasty (TKA), and high tibia osteotomy (HTO). ${ }^{[1-3]}$ The surgeries are often used for different patient groups; however, there is considerable overlap in the indications for all three options and it is often difficult to decide whether a UKA or TKA is more appropriate. ${ }^{[3-5]}$ Treatment of isolated compartmental osteoarthritis of the knee with UKA has mixed outcome and such procedure remains controversial. ${ }^{[6-9]}$ However, there has been a resurgence of interest and a major increase in the use of UKA for the treatment of medial compartmental knee osteoarthritis in the past decades. ${ }^{[6,7,10-13]}$ In the US market, UKA comprised approximately $1 \%$ of all knee arthroplasties in 1996 and 1997, and it increased to 6\% in 2000 and 2001. ${ }^{[14]}$ Improved prosthetic design, minimally invasive surgical techniques, and strict patient selection criteria have resulted in improved functional outcomes and survivorship rates. ${ }^{[15-22]}$ The advantages of UKA included the preservation of knee kinematics and good range of knee motion that may better suit the need of kneeling in activities of daily living in oriental countries. ${ }^{[6,20]}$ The purpose of this prospective study was to evaluate the functional outcomes of UKA for medial compartment knee arthropathy in Asian patients.

\section{METHODS}

The selection criteria of UKA included clinical, radiographic, and intraoperative assessments. ${ }^{[6,19]}$ The clinical criteria included pain and tenderness localized to medial joint line, knee flexion more than $90^{\circ}$, and fixed knee flexion deformity less than $10^{\circ}$. The radiographic criteria included isolated medial compartment osteoarthritis with complete loss of cartilage, osteonecrosis of medial femoral condyle, varus deformity less than $15^{\circ}$, and insignificant degenerative changes in other compartments. The intraoperative criteria included passively correctable varus deformity under anesthesia, intact anterior cruciate ligament, and full-thickness cartilage wear on the anteromedial half of the medial tibia plateau. The exclusion criteria included inflammatory arthritis, patellofemoral joint symptoms, hemophilia, chondrocalcinosis, symptomatic knee instability, body mass index (BMI) of greater than 32, prior HTO, full-thickness patellar cartilage loss, significant degenerative changes involving lateral or patellofemoral compartment, and severe angular deformity and flexion contracture. Forty-eight patients with 51 knees who met the above criteria and underwent UKA were included in this study. The patient demographic characteristics are shown in Table 1.

The surgical tips consisted of minimally invasive medial parapatellar approach with quadriceps spared, lateral displacement of patella, but not everted, avoidance of incidental fracture of the vertical tibial cut, minimal or none soft
Table 1: Patient demographic characteristics

\begin{tabular}{lc}
\hline Numbers of patients/knees & $48 / 51$ \\
Bilateral knees & 3 \\
Average age (years) (range) & $65.71 \pm 7.27(53-81)$ \\
Gender (female/male) & $35 / 13$ \\
Side (right/left) & $23 / 28$ \\
Diagnosis & \\
$\quad$ Osteoarthritis (medial compartment) & $82.4 \%(42 / 51)$ \\
Osteonecrosis (MFC) & $17.7 \%(9 / 51)$ \\
Average duration of disease (months) (range) & $18.3 \pm 15.23(12-74)$ \\
Average body weight (kg) (range) & $68.13 \pm 10.08(51-91)$ \\
Average BMI (body wt/body ht in \%) (range) & $28.31 \pm 3.75(22.6-31.4)$ \\
Average follow-up (months) (range) & $52.0 \pm 24.0(12-92)$ \\
\hline Abbreviations: MFC: Medial femoral condle; BMI: Body mass index
\end{tabular}

tissue release, equal flexion and extension gaps, replication of the tibia slope, centering of the femur component on the tibial component to reduce edge loading effect postoperatively and allow $2 \mathrm{~mm}$ medial laxity by undercorrection of $2^{\circ}-3^{\circ}$ of varus angulation. In this series, only one type of prosthesis (Zimmer, Warsaw, IN, USA) was used and all components were cemented in all cases.

Prophylaxis with broad-spectrum antibiotics was routinely given for $24 \mathrm{~h}$. However, prophylactic anticoagulation with low-molecular-weight heparin was given selectively in patients with high risk. Postoperative management included ambulation with weight bearing as tolerated, range of knee motion, and quadriceps and hamstring exercises starting from the second postoperative day. Patients were discharged from the hospital while independent on walking and continued outpatient physical therapy until full recovery.

The evaluation parameters included knee and functional scores of The Knee Society, subjective and objective scores of the International Knee Document Committee (IKDC), and radiographs of the operated knees. Functional evaluation included pain, alignment and range of motion of the knee, and ability to do kneeling or squatting for activities of daily living. Radiographic evaluation included the overall alignment of the knee, component position, and radiolucency at the prosthesis-bone interface.

\section{Statistical analysis}

The pre- and postoperative data were compared statistically using Wilcoxon signed-ranks test. The incidence of osteoarthritis in lateral and patellofemoral compartments was compared by Chi-square test. The statistical significance was set at $p<0.05$.

\section{RESULTS}

The Knee Society knee and functional scores and the IKDC scores before and after surgery are summarized in Table 2. Significant improvements were noticed in pain and function of the knee after UKA $(p<0.001)$. The over- 
all clinical results showed the functional outcome of the knee to be normal in 51\% (26 of 51), nearly normal in 37\% (19 of 51), abnormal in 8\% (4 of 51), and severely abnormal in $4 \%$ ( 2 of 51). The levels of functional activities included stair climbing in $96 \%$, squatting in $76 \%$, jogging in $71 \%$, and kneeling in $47 \%$. Approximately three-quarters of the patients were able to kneel for activities of daily living including religious service and customary cross-leg lifestyle. Approximately $98 \%$ of the patients were satisfied with the operation. The survivorship of the prosthesis was $98 \%$ at a follow-up of $52.0 \pm 24.0$ (range 12-92) months, with one revision pending.

The radiographic evaluation of the knee before and after surgery is summarized in Table 3. The femorotibial alignment was within normal physiological limits in all cases. Component position was centered in $82 \%$ and off-centered in $18 \%$. Osteoarthritis was $22 \%$ preoperative and $27 \%$ postoperative for the patellofemoral compartment, and $0 \%$ before and $4 \%$ after surgery for the lateral compartment. The functional activities of patients with and without patellofemoral arthritis are summarized in Table 4 . The functional outcomes showed no differences between patients with and

Table 2: Functional score, IKDC score, and range of knee motion

\begin{tabular}{lccc}
\hline & Preoperative & Postoperative & $p$ value $^{\text {a }}$ \\
\hline Functional & $51.1 \pm 18.6$ & $86.8 \pm 12.3$ & $<0.001$ \\
score (range) & $(25-75)$ & $(60-100)$ & \\
IKDC (range) & $27.0 \pm 10.7$ & $79.5 \pm 19.4$ & $<0.001^{\text {a }}$ \\
& $(15-50)$ & $(35-100)$ & \\
Range of knee & $115^{\circ} \pm 5.05^{\circ}$ & $125^{\circ} \pm 10.5^{\circ}$ & $<0.001^{\text {a }}$ \\
motion (range) & $\left(0^{\circ}-125^{\circ}\right)$ & $(0-145)$ & \\
\hline
\end{tabular}

aThe $p$ values are obtained by Wilcoxon signed-ranks test.

Abbreviation: IKDC: International knee document committee

Table 3: The radiographic evaluation

\begin{tabular}{|c|c|c|c|}
\hline Femorotibial alignment & Preoperative & Postoperative & $p$ value \\
\hline Average (range) & $\begin{array}{c}177.27 \pm 2.23 \\
(170.7-179.9)\end{array}$ & $\begin{array}{c}175.0 \pm 2.43 \\
(174.1-179.2)\end{array}$ & $<0.001^{\mathrm{a}}$ \\
\hline Tibial slope (range) & $\begin{array}{c}85.5^{\circ} \pm 6.46^{\circ} \\
\left(74.7^{\circ}-97.9^{\circ}\right)\end{array}$ & $\begin{array}{c}88.19^{\circ} \pm 4.42^{\circ} \\
\left(77^{\circ}-97.1^{\circ}\right)\end{array}$ & $<0.001^{\mathrm{a}}$ \\
\hline Patellar tilt & $12 \%$ & $13 \%$ & $1.0^{\mathrm{b}}$ \\
\hline Radiolucency & - & $2 \%(1 / 51)$ & \\
\hline \multicolumn{4}{|l|}{ Component position } \\
\hline Center & & $82 \%(42 / 51)$ & \\
\hline $\begin{array}{l}\text { Medial } \\
\text { (femur on tibia) }\end{array}$ & & $\begin{array}{c}16 \%(8 / 51) \text { (average } \\
2.18 \pm 0.55 \mathrm{~mm}), \\
\text { (range } 1.35-3.0 \mathrm{~mm} \text { ) }\end{array}$ & \\
\hline $\begin{array}{l}\text { Lateral } \\
\text { (femur on tibia) }\end{array}$ & & $2 \%(1 / 51)(3.5 \mathrm{~mm})$ & \\
\hline \multicolumn{4}{|l|}{ Degenerative changes } \\
\hline Lateral compartment & $0 \%$ & $4 \%(2 / 51)$ & $0.125^{\mathrm{b}}$ \\
\hline $\mathrm{P} / \mathrm{F}$ compartment & $22 \%(11 / 51)$ & $27 \%(14 / 51)$ & $0.250^{\mathrm{b}}$ \\
\hline Cement protrusion & $9.8 \%(5 / 51)$ & & \\
\hline
\end{tabular}

${ }^{\text {a The }} p$-values are obtained statistically by Wilcoxon signed-ranks test.

${ }^{\mathrm{b}}$ The $p$-values are obtained by McNemar's test without patellofemoral arthritis pre- and postoperatively. Radiolucency was noted in only one case $(2 \%)$ and was non-progressive. Cement protrusion was identified in $9.8 \%$ and none was symptomatic. The pre- and postoperative anteroposterior (AP) radiographs of the knee at 1, 4, and 6 years are shown in Figure 1.

Complications included no infection, no deep vein thrombosis (DVT), one component malposition, and one knee pain of undetermined origin.

\section{DISCUSSION}

The initial results reported UKA to be unfavorable in the early stage of development. ${ }^{[8,9]}$ However, there have been big changes with more favorable results in the past decades. The survivorship of UKA was reported to be between $96 \%$ and $98 \%$ at $7-10$ years. ${ }^{[6,13,19]}$ This was attributed to strict patient selection, improved prosthetic design, and minimally invasive surgery. ${ }^{[7,13,21-25]}$ In the present study, the functional outcome of the knee was normal in 51\%, nearly normal in $37 \%$, abnormal in $8 \%$, and severely abnormal in $4 \%$. Approximately $98 \%$ of the patients were satisfied with the surgery. The survivorship of the prosthesis was $98 \%$ at a follow-up of $52.0 \pm 24.0$ (range 12-92) months when the case of pending revision was counted as failure.

Several studies reported nearly normal gait with preserved biphasic pattern and similar kinematic profiles of normal knee after UKA. ${ }^{[26-28]}$ The gait velocity and muscle strength after UKA are superior to those after HTO..$^{[2,27,28]}$ UKA provided improved range of motion, decreased

Table 4: Patellofemoral arthritis

\begin{tabular}{|c|c|c|c|}
\hline & Preoperative & Postoperative & $p$-value ${ }^{1}$ \\
\hline With P/F OA & $22 \%(11 / 51)$ & $27 \%(14 / 51)$ & 0.001 \\
\hline Without P/F OA & $78 \%(40 / 51)$ & $73 \%(37 / 51)$ & $<0.001$ \\
\hline$p$-value ${ }^{2}$ & $<0.001$ & $<0.001$ & \\
\hline \multicolumn{4}{|l|}{ Function score } \\
\hline With P/F OA & $52.6 \pm 16.6(25-71)$ & $92.6 \pm 10.37(69-100)$ & 0.028 \\
\hline Without P/F OA & $53.9 \pm 18.8(10-75)$ & $87.0 \pm 12.2(60-100)$ & $<0.001$ \\
\hline$p$-value ${ }^{2}$ & 0.286 & 0.225 & \\
\hline \multicolumn{4}{|l|}{ Kneeling } \\
\hline With P/F OA & $18 \%(2 / 11)$ & $79 \%(11 / 14)$ & 0.004 \\
\hline Without P/F OA & $20 \%(8 / 40)$ & $81 \%(30 / 37)$ & $<0.001$ \\
\hline$p$-value ${ }^{2}$ & 0.633 & 0.561 & \\
\hline \multicolumn{4}{|l|}{ Squatting } \\
\hline With P/F OA & $18 \%(2 / 11)$ & $71 \%(10 / 14)$ & 0.008 \\
\hline Without P/F OA & $15 \%(6 / 40)$ & $70 \%(26 / 37)$ & $<0.001$ \\
\hline$p$-value ${ }^{2}$ & 0.557 & 0.611 & \\
\hline \multicolumn{4}{|l|}{ Sit to stand } \\
\hline With P/F OA & $27 \%(3 / 11)$ & $100 \%(14 / 14)$ & 0.008 \\
\hline Without P/F OA & $23 \%(9 / 40)$ & $97 \%(36 / 37)$ & $<0.001$ \\
\hline$p$-value ${ }^{2}$ & 0.706 & 0.725 & \\
\hline
\end{tabular}

P/F OA: Patellofemoral arthritis; ${ }^{1} p$-value: Comparison of preoperative and postoperative data within the same group; ${ }^{2} p$-value: Comparison of data between two groups

Biomed J Vol. 37 No. 6

November - December 2014 

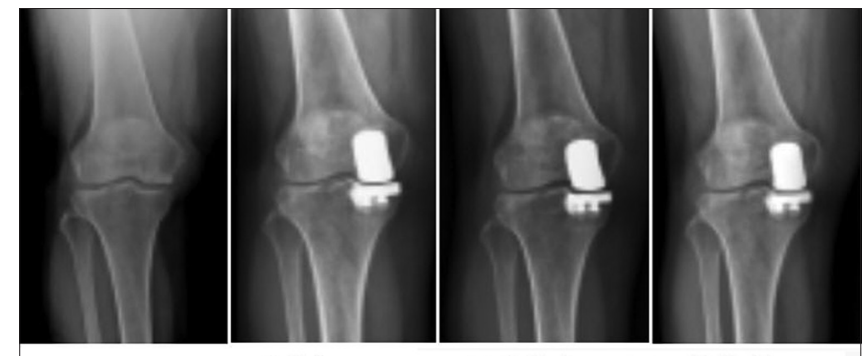

Pre-op

P. 0.1 year.

P.O. 4 years.

P.O. 6 years.

Figure 1: The pre- and postoperative AP radiographs of the knee at 1 , 4 , and 6 years showed centered UKA components in normal alignment and no radiolucency.

rehabilitation time, and immediate weight bearing than HTO. However, HTO showed ability to maintain a higher level of activity without potential wear of arthroplasty components. Overall, UKA showed slightly better results in survival and functional outcome..$^{[1,2,20]}$ The majority of patients are able to return to recreational sports involving low- and mid-impact activities after UKA. ${ }^{[29,30]}$ In this study, the levels of functional activities of the knee included stair climbing in $96 \%$, squatting in $76 \%$, jogging in $71 \%$, and kneeling in $47 \%$. Approximately three-quarters of the patients are able to perform normal activities of daily living such as religious service and cross-leg sitting lifestyle.

One concern of UKA is the progression of osteoarthritic changes in the lateral and patellofemoral compartments and ultimately necessitated TKA. Progression of osteoarthritis to the lateral compartment was reported in $18 \%$ patients and to the patellofemoral compartment in $14 \%$ patients in 10 years. ${ }^{[6,31]}$ The cumulative revision rate for UKA is $22 \%$ for patients younger than 60 years which is in part due to the progressive degenerative changes of other compartments. ${ }^{[32]}$ UKA is reserved for knees with isolated uni-compartmental osteoarthritis of the knee. However, early osteoarthritis of the patellofemoral joint with medial spur formation, but very subtle cartilage fissuring that is difficult to identify in clinical assessment and radiographic examination can only be verified intraoperatively. Under this circumstance, UKA and concomitant exostectomy and debridement of the patellofemoral joint were performed instead of TKA. In the present study, osteoarthritis of the patellofemoral joint was $22 \%$ before and $27 \%$ after surgery, and osteoarthritis of the lateral compartment was 0 before and $4 \%$ after surgery. The clinical outcomes were not affected when concomitant surgery was performed in the patellofemoral joint. Therefore, based on the results of the present study, medial compartmental osteoarthritis combined with early patellofemoral arthritis is not a contraindication for UKA. Some reports showed higher patient satisfaction after revision of a failed UKA than after revision of a failed TKA, and more surgeons recom- mend UKA because of functional superiority. ${ }^{[33]}$ In this study, there was no revision or conversion of UKA to TKA due to progressive osteoarthritis of the knee at an average follow-up $52.0 \pm 24.0$ months.

The complications associated with TKA, such as infection, DVT, and stiffness, are rare after UKA. ${ }^{[21,24,31]}$ In the present series, the complications included no infection, no DVT, one knee pain due to component malposition on X-ray, and one knee pain of undetermined origin.

This study has its own limitations. It is limited by virtue of the small number of patient population which may result in bias due to low power of statistical analysis. The average follow-up time was approximately 4 years, and longer follow-up is needed to ascertain the ultimate results of UKA in Asian patients. Other limitations include lack of control group in this study. The patients selected included those with a mixture of osteoarthritis and osteonecrosis of the medial compartment of the knee, and no stratification between the two diagnoses was performed. This study did not stratify patients younger than 60 years from the cohort population. The purpose of this study was not to compare the functional results of UKA with other procedures such as TKA or HTO, but to evaluate the functional results of UKA in Asian patients.

\section{Conclusion}

UKA provided excellent pain relief and restored the knee function in Asian patients with medial compartment knee arthropathy with a high rate of patient satisfaction at medium-term follow-up. Approximately three-quarters of the patients are able to perform normal activities of daily living including kneeling for religious service and cross-leg sitting lifestyle. Long-term follow-up is needed to further ascertain the ultimate outcome of UKA in Asian patients.

\section{Acknowledgments}

No benefits in any form have been received or will be received from a commercial party related directly or indirectly to the subject of this article.

\section{REFERENCES}

1. Dettoni F, Bonasia DE, Castoldi F, Bruzzone M, Blonna D, Rossi R. High tibial osteotomy versus unicompartmental knee arthroplasty for medial compartment arthrosis of the knee: A review of the literature. Iowa Orthop J 2010;30:131-40.

2. Ivarsson I, Gillquist J. Rehabilitation after high tibial osteotomy and unicompartmental arthroplasty. A comparative study. Clin Orthop Relat Res 1991;266:139-44.

3. RougraffBT, Heck DA, Gibson AE. A comparison of tricompartmental and unicompartmental arthroplasty for the treatment of gonarthrosis. Clin Orthop Relat Res 1991;273:157-64. 
4. Newman JH, Ackroyd CE, Shah NA. Unicompartmental or total knee replacement? Five-year results of a prospective, randomised trial of 102 osteoarthritic knees with unicompartmental arthritis. J Bone Joint Surg Br 1998;80:862-5.

5. Saldanha KA, Keys GW, Svard UC, White SH, Rao C. Revision of Oxford medial unicompartmental knee arthroplasty to total knee arthroplasty-Results of a multicentre study. Knee 2007;14:275-9.

6. Berger RA, Meneghini RM, Jacobs JJ, Sheinkop MB, Della Valle CJ, Rosenberg AG, et al. Results of unicompartmental knee arthroplasty at a minimum of ten years of follow-up. J Bone Joint Surg Am 2005;87:999-1006.

7. Emerson RH Jr, Higgins LL. Unicompartmental knee arthroplasty with the Oxford prosthesis in patients with medial compartment arthritis. J Bone Joint Surg Am 2008;90:118-22.

8. Insall J, Walker P. Unicondylar knee replacement. Clin Orthop Relat Res 1976;20:83-5.

9. Laskin R. Unicompartmental tibiofemoral resurfacing arthroplasty. J Bone Joint Surg Am 1978;60:182-5.

10. Koskinen E, Paavolainen P, Eskelinen A, Harilainen A, Sandelin J, Ylinen $\mathrm{P}$, et al. Medial unicompartmental knee arthroplasty with Miller-Galante II prosthesis: Mid-term clinical and radiographic results. Arch Orthop Trauma Surg 2009;129:617-24.

11. Mercier N, Wimsey S, Saragaglia D. Long-term clinical results of the Oxford medial unicompartmental knee arthroplasty. Int Orthop 2010;34:1137-43.

12. Murray DW, Goodfellow JW, O'Connor JJ. The Oxford medial unicompartmental arthroplasty: A ten-year survival study. J Bone Joint Surg Br 1998;80:983-9.

13. Parmaksizoglu AS, Kabukcuoglu Y, Ozkaya U, Bilgili F, Aslan A. Short-term results of the Oxford phase 3 unicompartmental knee arthroplasty for medial arthritis. Acta Orthop Traumatol Turc 2010;44:135-42.

14. US markets for reconstructive devices 2002. Toronto: Millennum Research Group; 2003.

15. Cinar BM, Akpinar S, Uysal M, Cesur N, Hersekli MA, Ozalay M, et al. Unicondylar knee arthroplasty in medial unicompartmental osteoarthritis: Technical faults and difficulties. Eklem Hastalik Cerrahisi 2010;21:31-7.

16. Kaya Bicer E, Servien E, Lustig S, Demey G, Ait Si Selmi T, Neyret P. Sagittal flexion angle of the femoral component in unicompartmental knee arthroplasty: Is it same for both medial and lateral UKAs? Knee Surg Sports Traumatol Arthrosc 2010;18:928-33.

17. Kim KT, Lee S, Cho KH, Kim KS. Fracture of the medial femoral condyle after unicompartmental knee arthroplasty. J Arthroplasty 2009;24:1143.e21-4.

18. Luscombe KL, Lim J, Jones PW, White SH. Minimally invasive Oxford medial unicompartmental knee arthroplasty. A note of caution. Intern Orthop 2007;31:321-4.
19. Pandit H, Jenkins C, Barker K, Dodd CA, Murray DW. The Oxford medial unicompartmental knee replacement using a minimally-invasive approach. J Bone Joint Surg Br 2006;88:54-60.

20. Price AJ, Dodd CA, Svard UG, Murray DW. Oxford medial unicompartmental knee arthroplasty in patients younger and older than 60 years of age. J Bone Joint Surg Br 2005;87:1488-92.

21. Daniilidis K, Skwara A, Skuginna A, Fischer F, Tibesku CO. Comparison of medium-term clinical and radiological outcome between cemented and cementless medial unicompartmental knee arthroplasty. Z Orthop Unfall 2009;147:188-93.

22. Song MH, Kim BH, Ahn SJ, Yoo SH, Lee MS. Early complications after minimally invasive mobile-bearing medial unicompartmental knee arthroplasty. J Arthroplasty 2009;24:1281-4.

23. Jenny JY. Comparative study of the use of computer assisted navigation system for axial correction in medial unicompartmental knee arthroplasty. Knee Surg Sports Traumatol Arthrosc 2010;18:1297.

24. Lim MH, Tallay A, Bartlett J. Comparative study of the use of computer assisted navigation system for axial correction in medial unicompartmental knee arthroplasty. Knee Surg Sports Traumatol Arthrosc 2009; 17:341-6.

25. Rudol G, Jackson MP, James SE. Medial tibial plateau fracture complicating unicompartmental knee arthroplasty. J Arthroplasty 2007;22:148-50.

26. Webster KE, Wittwer JE, Feller JA. Quantitative gait analysis after medial unicompartmental knee arthroplasty for osteoarthritis. J Arthroplasty 2003;18:751-9.

27. Hollinghurst D, Stoney J, Ward T, Gill HS, Newman JH, Murray DW, et al. No deterioration of kinematics and cruciate function 10 years after medial unicompartmental arthroplasty. Knee 2006;13:440-4.

28. Patil S, Colwell CW Jr, Ezzet KA, D'Lima DD. Can normal knee kinematics be restored with unicompartmental knee replacement? J Bone Joint Surg Am 2005;87:332-8.

29. Fisher N, Agarwal M, Reuben SF, Johnson DS, Turner PG. Sporting and physical activity following Oxford medial unicompartmental knee arthroplasty. Knee 2006;13:296-300.

30. Naal FD, Fischer M, Preuss A, Goldhahn J, von Knoch F, Preiss S, et al. Return to sports and recreational activity after unicompartmental knee arthroplasty. Am J Sports Med 2007;35:1688-95.

31. Ansari S, Warwick D, Ackroyd CE, Newman JH. Incidence of fatal pulmonary embolism after 1,390 knee arthroplasties without routine prophylactic anticoagulation, except in high-risk cases. J Arthroplasty 1997;12:599-602.

32. Harrysson OL, Robertsson O, Nayfeh JF. Higher cumulative revision rate of knee arthroplasties in younger patients with osteoarthritis. Clin Orthop Relat Res 2004;421:162-8.

33. Robertsson O, Dunbar M, Pehrsson T, Knutson K, Lidgren L. Patient satisfaction after knee arthroplasty: A report on 27,372 knees operated on between 1981 and 1995 in Sweden. Acta Orthop Scand 2000;71:262-7. 